\title{
Synthesis of Indoles and Benzisoxazolines from Baylis-Hillman Adducts of 2-Nitrobenzaldehydes
}

\author{
Ka Young Lee, Hyun Seung Lee, and Jae Nyoung Kim \\ Department of Chemistry and Institute of Basic Science, Chomam Mational Lniversity, Gwangiu 500-757, Korea \\ E-mail: kiminachonnamackr \\ Received October 17, 2006
}

Key Words : Indoles. Baylis-Hillman adducts. 2-Nitrobenzaldehydes. $\mathrm{SnCl}_{2}$

Recently a variety of interesting chemical transformations involving the use of Baylis-Hillman adducts have been reported. ${ }^{1-4}$ Among them the use of Baylis-Hillman adducts of 2-nitrobenzaldehydes have been investigated extensively

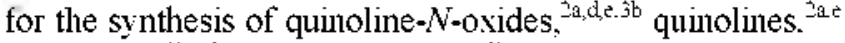
quinolones. ${ }^{\text {tbr. }, 3 \mathrm{a} a}$ benzisoxazolines, ${ }^{\text {tb }}$ and indoles. ${ }^{4}$ Although there have been reported numerous methods for the synthesis of indole derivatives, ${ }^{4.6}$ a development of new synthetic method of indole scaffold is required due to their biological importance and usefulness as synthetic intermediates. $^{4}$

A few years ago we reported the synthesis of 3-alkoxymethyl $2(l H)$-quinolones from the $\mathrm{SnCl}_{2}$-mediated reduction of the Baylis-Hillman adducts 1a in alcohol solvent (Scheme 1). ${ }^{\text {b }}$ In the reaction. the nitro group of $1 \mathrm{a}$ was reduced to amino group and we obtained 3-alkoxymethyl $2(l H)$-quinolones as the major products. ${ }^{-b}$ In some cases we obtained benzisoxazoline compounds (5-10\%) like $3 \mathrm{a}$ as the side products. which might be formed wa the hydroxylamine intermediate. ${ }^{\text {tb }}$ At that time we also observed the formation of trace amounts of indole derivatives in some cases. especially when we used alcohols having high boiling point. $^{7}$

Encouraged by recent publications on the synthesis of indoles from Baylis-Hillman adducts. ${ }^{4}$ we reinvestigated the $\mathrm{SnCl}_{\mathbf{2}}$-mediated reduction of $\mathbf{1 a}$ in order to obtain the benzisoxazoline or indole derivatives in improved yields. To our delight, we found an efficient condition for the formation of 3-substituted indoles and benzisoxazolines and wish to report herein the results (Scheme 1).

As shown in Scheme 1, the reaction of Baylis-Hillman adduct 1a of 2-nitrobenzaldehyde and $\mathrm{SnCl}_{2}$ in 1,4-dioxane at refluxing temperature gave the indole $2 \mathrm{a}$ and benzisoxazoline 3a in moderate yields. ${ }^{\circ}$ As described above the formation of benzisoxazoline compound was already reported in part in our previous paper ${ }^{b}$ and the reaction mechanism could be regarded involving the lydroxylamine intermediate (I) and the cyclized intermediate (II) as in Scheme 2 .

Although the reaction mechanism for the formation of indole 2a was not clear at this stage we could tentatively propose a plausible mechanism as shown in Scheme 2: (i) formation of nitroso internnediate (III). (ii) Michael addition to form (IV). (iii) reversible retro-aldol type ring-opening to form (V). (iv) cyclization to (VI). (v) water-assisted elimination of formic acid to give indoline derivative (VII), (vi) delydration to (VIII) and the funal isomerization to indole 2a.

Based on the experimental results and the proposed mechanism there must be formed hydroxylamine intermediate (I) and nitroso intermediate (III) under $\mathrm{SnCl}_{2} /$ dioxane conditions in a variable ratios. In order for the selective formation of either (I) or (III). we exammed the reaction conditions including solvent. temperature, the equivalents of $\mathrm{SnCl}_{2}$, but all failed to improve the yields or selectivity. The nitro group is readily converted to a series of functions of various degrees of reduction: very exceptionally to a nitroso group. more often to a hydroxylamino group and most frequently to the amino group ${ }^{2 e .9}$ Nitroso compounds are usually not obtained directly but rather by reoxidation of hydroxylamino compounds, which can be prepared from nitro compounds by $\mathrm{SnCl}_{2}{ }^{2.9}$ Moreover there have been few instances in

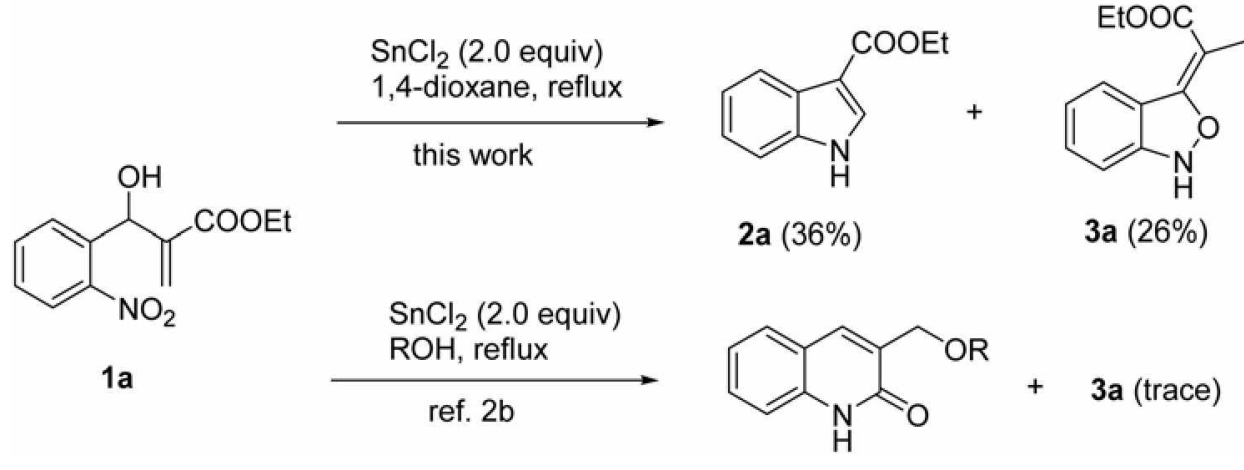

Scheme 1 


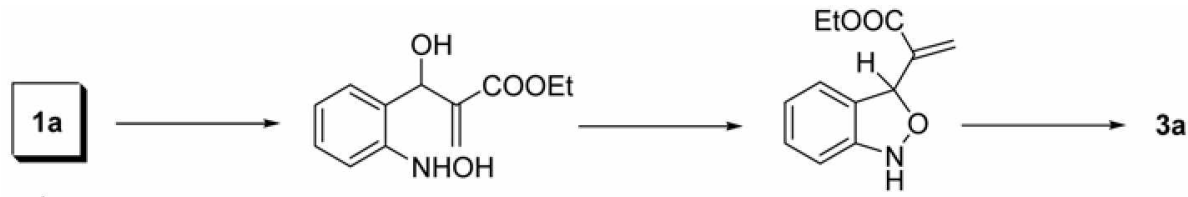

(I)

(II)<smiles>C=C(C(=O)OCC)C(O)c1ccccc1N=O</smiles>

(III)<smiles>CCO/C(O)=C1\C[N+](=O)c2ccccc2C1[O-]</smiles>

(IV)<smiles>CCO/C([O-])=C/CN(C)c1ccccc1C=O</smiles>

(V)

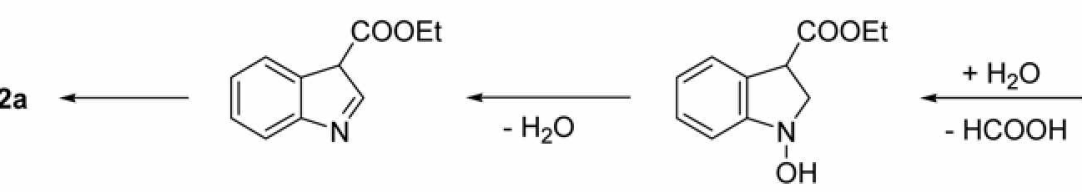

(VIII)<smiles>CCOC(=O)C1C[N+]([O-])=C2C=CC=CC21C(=O)O</smiles>

( $\mathbf{V} \mathbf{l}$

Scheme 2

Table 1. Synthesis of indoles and benzisoxazolines ${ }^{a}$<smiles>CCOC(=O)C(C)=C1ONc2cc(Cl)ccc21</smiles>

"Conditions: 1 (1.0 equiv), dioxane. $\mathrm{SnCl}$ ( 2.0 equiv), reflux. "Not tormed. which nitroso compounds have been isolated as intermediates in reductions of nitro compounds. It is interesting to compare the results with our previous paper. ${ }^{2 t}$ In our pervious paper we also proposed the involvement of nitroso intermediate. which was converted into quinoline $N$-oxides via Michael addition followed by dehydration. ${ }^{2 c}$ Delydration occurred efficiently in the acidic conditions. However. when we used $\mathrm{SnCl}_{2} /$ dioxane conditions, retro-aldol type ringopening reaction of the zwitterinoic intermediate (IV) proceeded as in Scheme 2 instead of dehydration. Similarly. Baylis-Hillman adducts $\mathbf{1 b}$ and $\mathbf{1 c}$ gave similar results as shown in Table 1. We also prepared indole derivatives 2 d-f from the reactions of $\mathbf{1 d - f}$ in moderate yields (44-48\%). However. we could not observe the corresponding benzisoxazline derivatives $\mathbf{3 d - f}$ in these cases on TLC. unexpectedly:

Whatever the details of the mechanism, the reaction represents an unprecendented. reproducible, and useful method for the preparation of interesting indoles and benzisoxazolines starting from the readily available Baylis-Hillman adducts.

\section{Experimental Section}

Typical procedure for the synthesis of $2 a$ and $3 a$ : To a stirred mixture of $1 \mathrm{a}(251 \mathrm{mg} .1 .0 \mathrm{mmol})$ in dioxane $(4 \mathrm{~mL})$ was added tin (II) chloride dihydrate $(451 \mathrm{mg} .2 .0 \mathrm{mmol}$ ) and the reaction mixture was heated to reflux for $10 \mathrm{~h}$. The reaction mixture was cooled to room temperature and poured into cold saturated $\mathrm{NH}_{4} \mathrm{Cl}$ solution. After extraction with EtOAc. washing with aq $\mathrm{K}_{2} \mathrm{CO}_{3}$ solution, and column chromatographic purification process (hexanes/EtOAc, 5:1) we obtained $2 \mathrm{a}(68 \mathrm{mg} .36 \%$ ) and $3 \mathrm{a}(57 \mathrm{mg} .26 \%$ ) as white solids. The representative spectroscopic data of prepared compounds are as follows. 
Compound 2a: ${ }^{\mathrm{a}, \mathrm{b}} 36 \%$ : white solid. mp $125-126^{\circ} \mathrm{C}$ : IR (KBr) 3271. 1668. 1531.1435 $\mathrm{cm}^{-1}$ : ${ }^{1} \mathrm{H}$ NMR $(300 \mathrm{MHz}$. $\left.\mathrm{CDCl}_{3}\right) \delta 1.46(\mathrm{t}, J=7.2 \mathrm{~Hz}, 3 \mathrm{H}) .4 .41$ (q. $J=7.2 \mathrm{~Hz}, 2 \mathrm{H}$ ). 7.25-7.31 (m. 2H). 7.40-7.44 (m. 1H). $7.93(\mathrm{~d} . J=3.0 \mathrm{~Hz}$. lH) $8.18-8.21(\mathrm{~m} .1 \mathrm{H}) .8 .68($ br s. $1 \mathrm{H}):{ }^{13} \mathrm{C}$ NMR $(75 \mathrm{MHz}$. $\left.\mathrm{CDCl}_{3}\right) \delta 14.56 .59 .85 .109 .07,111.54,121.54,121.99$. 123.14. 125.83, 131.06, 136.16, 165.43. Anal. Calcd for $\mathrm{C}_{11} \mathrm{H}_{11} \mathrm{NO}_{2}:$ C. 69.83: H. 5.86: N. 7.40. Found: C. 69.58: H. 5.92: N. 7.32

Compound 3a: $26 \%$ : white solid, mp $187-188{ }^{\circ} \mathrm{C}$ : IR (KBr) $3305.1662 .1200 \mathrm{~cm}^{-1}:{ }^{1} \mathrm{H}$ NMR $\left(300 \mathrm{MHz}, \mathrm{CDCl}_{3}\right)$ $\delta 1.45$ (t. $J=7.2 \mathrm{~Hz}, 3 \mathrm{H}), 2.75$ (s. $3 \mathrm{H}), 4.40$ (q. $J=7.2 \mathrm{~Hz}$. $2 \mathrm{H}) .7 .18-7.32(\mathrm{~m} .3 \mathrm{H}) .8 .08-8.12(\mathrm{~m} .1 \mathrm{H}) .8 .31(\mathrm{br} \mathrm{s} . \mathrm{HH})$ : ${ }^{13} \mathrm{C} \mathrm{NMR}\left(75 \mathrm{MHz} . \mathrm{CDCl}_{3}\right) \delta 14.23 .14 .59,59.47 .104 .71$. $110.40,121.34 .121 .67,122.33,127.16,134.41,143.82$. 166.06. Anal. Calcd for $\mathrm{C}_{1=} \mathrm{H}_{3} \mathrm{NO}_{3}: \mathrm{C}, 65.74 ; \mathrm{H}, 5.98: \mathrm{N}$. 6.39. Found: C. $65.65:$ H. $5.91:$ N. 6.27 .

Compound 2b: $41 \%$ : white solid $m p$ 126-127 ${ }^{\circ} \mathrm{C}$ : IR (KBr) 3288, 1695, 1419. $1174 \mathrm{~cm}^{-1}$ : ${ }^{1} \mathrm{H}$ NMR $(300 \mathrm{MHz}$. $\left.\mathrm{CDCl}_{3}+\mathrm{DMSO}_{-} \mathrm{d}_{6}\right) \delta \mathrm{l} .40$ (t. $\left.J=7.2 \mathrm{~Hz}, 3 \mathrm{H}\right) .4 .38$ (q. $J=$ $7.2 \mathrm{~Hz}, 2 \mathrm{H}$ ). $7.13-7.33$ (m. 3H). 7.92 (d. $J=3.0 \mathrm{~Hz} . \mathrm{lH}$ ). 8.84 (br s. $1 \mathrm{H}$ ): ${ }^{13} \mathrm{C}$ NMR $\left(75 \mathrm{MHz} . \mathrm{CDCl}_{3}+\mathrm{DMSO}_{-1}\right.$ ) $\delta$ 14.43,60.32, 109.80, 110.31. 122.99, 123.77, 123.81. 126.85. 132.23. 137.96. 164.08. Anal. Calcd for $\mathrm{C}_{11} \mathrm{H}_{10} \mathrm{ClNO}_{2}: \mathrm{C}$. 59.07: H. 4.51: N. 6.26. Found: C. 59.22: H. 4.63: N. 6.08 .

Compound 3b: $25 \%$ : white solid mp 147-148 ${ }^{\circ} \mathrm{C}$ : IR (KBr) 3298. 1693. $1435 \mathrm{~cm}^{-1}$. ${ }^{1} \mathrm{H}$ NMR $\left(300 \mathrm{MHz}, \mathrm{CDCl}_{3}+\right.$ DMSO-d 6$) \delta 1.42(\mathrm{t} . J=7.2 \mathrm{~Hz}, 3 \mathrm{H}) .2 .62(\mathrm{~s}, 3 \mathrm{H}) .4 .40(\mathrm{q} . J$ $=7.2 \mathrm{~Hz} .2 \mathrm{H}) .7 .03-7.23(\mathrm{~m} .3 \mathrm{H}), 9.60($ br s. $1 \mathrm{H}) .{ }^{13} \mathrm{C}$ NMR $\left(75 \mathrm{MHz}, \mathrm{CDCl}_{3}+\mathrm{DMSO}_{6}\right) \quad \delta 13.78,14.42 .60 .22$. 109.46. 122.43, 122.51, 122.91, 124.02, 125.65, 136.53. 142.42. 165.67. Anal. Caled for $\mathrm{C}_{12} \mathrm{H}_{1}=\mathrm{ClNO}_{3}: \mathrm{C} .56 .81 ; \mathrm{H}$. 4.77: N. 5.52. Found: C. 56.49: H. 4.48: N. 5.39

Compound $2 \mathrm{c}: 38 \%$; white solid. mp $186-187^{\circ} \mathrm{C}$ : IR (KBr) 3228. 1666. 1446. $1190 \mathrm{~cm}^{-1}:{ }^{1} \mathrm{H}$ NMR $(300 \mathrm{MHz}$. $\left.\mathrm{CDCl}_{3}+\mathrm{DMSO}_{-} \mathrm{d}_{6}\right) \delta \mathrm{l} .32$ (t. $J=7.2 \mathrm{~Hz}, 3 \mathrm{H}$ ). 4.29 (q. $J=$ $7.2 \mathrm{~Hz}, 2 \mathrm{H}) .7 .08-7.31$ (m. 2H). 7.85 (d. $J=3.0 \mathrm{~Hz}, \mathrm{lH}$ ). 8.00 (s. $1 \mathrm{H}) .11 .40$ (br s. $1 \mathrm{H}):{ }^{13} \mathrm{C}$ NMR $\left(75 \mathrm{MHz} . \mathrm{CDCl}_{3}+\right.$ DMSO-d 6$) \delta 13.89,58.83,106.77 .112 .58,119.79 .121 .99$. 126.29. 126.40, 132.14. 134.36. 164.13.

Compound $3 \mathrm{c}: 26 \%$ : white solid mp $168-169^{\circ} \mathrm{C}$ : IR (KBr) 3277. $1666 \mathrm{~cm}^{-1}:{ }^{1} \mathrm{H}$ NMR $\left(300 \mathrm{MHz}, \mathrm{CDCl}_{3}+\right.$ DMSO-d 6$) \delta 1.45(\mathrm{t} . J=7.2 \mathrm{~Hz}, 3 \mathrm{H}) .2 .74(\mathrm{~s}, 3 \mathrm{H}) .4 .40(\mathrm{q} . J$ $=7.2 \mathrm{~Hz} .2 \mathrm{H}), 7.14(\mathrm{dd} . J=8.4$ and $1.8 \mathrm{~Hz}, 1 \mathrm{H}) .7 .2 \mathrm{l}(\mathrm{d}, J=$ $8.4 \mathrm{~Hz}, 1 \mathrm{H}) .8 .07(\mathrm{~d} J=1.8 \mathrm{~Hz}, \mathrm{lH}) .8 .46\left(\right.$ br s. $1 \mathrm{H}$ ): ${ }^{13} \mathrm{C}$ NMR $\left(75 \mathrm{MHz}, \mathrm{CDCl}_{\mathrm{j}}+\mathrm{DMSO}_{-}\right) \delta 1+12.14 .62 .59 .32$. 103.50. 111.95. 120.40, 121.81. 126.70. 128.43, 133.41 . 145.99. 165.75

Compound 2d: ${ }^{6} 48 \%$; white solid, mp $177-178{ }^{\circ} \mathrm{C}$ : IR (KBr) $3251,2226,1431 \mathrm{~cm}^{-1} ;{ }^{1} \mathrm{H}$ NMR $(300 \mathrm{MHz}$, DMSO$\left.\mathrm{d}_{6}\right) \delta 7.20-7.3 \mathrm{l}(\mathrm{m} .2 \mathrm{H}) .7 .56-7.65(\mathrm{~m}, 2 \mathrm{H}), 8.24(\mathrm{~s} . \mathrm{HH})$. $12.2 \mathrm{l}$ (br s. $\mathrm{lH}$ ): ${ }^{13} \mathrm{C}$ NMR $\left(75 \mathrm{MHz}, \mathrm{CDCl}_{3}+\mathrm{DMSO}_{-1}\right) \delta$ $84.33,113.04,116.46,118.51,121.78$. 123.47. 126.85 . 134.57. 135.33: Mass $(70 \mathrm{eV}) \mathrm{mz}$ (rel. intensity) 88 (7). 115 (30). $142\left(\mathrm{M}^{+} .100\right)$

Compound 2e: $48 \%$; white solid, mp $223-225^{\circ} \mathrm{C}$ (dec.):
IR (KBr) $3263,2224 \mathrm{~cm}^{-1}$ : ${ }^{1} \mathrm{H}$ NMR $(300 \mathrm{MHz}$, DMSO-d $)$ $\delta 7.26-7.28(\mathrm{~m}, 2 \mathrm{H}) .7 .50-7.56(\mathrm{~m} . \mathrm{HH}) .8 .39(\mathrm{~s} . \mathrm{HH}) .12 .52$ (br s. $1 \mathrm{H}):{ }^{13} \mathrm{C}$ NMR $\left(75 \mathrm{MHz}, \mathrm{DMSO}-\mathrm{d}_{\text {a }}\right) \delta 83.56,112.11$, $116.13 .121 .78,123.40,124.14,124.24,136.36,136.59$.

Compound 2f: $4 \%$ : white solid. mp $198-199^{\circ} \mathrm{C}$ : IR (KBr) 3273, 2224, 1415, $1304 \mathrm{~cm}^{-1} ;{ }^{1} \mathrm{H}$ NMR $(300 \mathrm{MHz}$, DMSO-d $s) \delta 7.30(\mathrm{dd}, J=8.7$ and $2.1 \mathrm{~Hz} . \mathrm{lH}), 7.58(\mathrm{~d} . J=$ $8.7 \mathrm{~Hz} .1 \mathrm{H}) .7 .67$ (d. $J=2.1 \mathrm{~Hz} .1 \mathrm{H}) .8 .30(\mathrm{~s} .1 \mathrm{H}) .12 .38(\mathrm{br}$ s. $1 \mathrm{H}):{ }^{13} \mathrm{C}$ NMR $\left(75 \mathrm{MHz}, \mathrm{DMSO}-\mathrm{d}_{6}\right) \delta 84.03,114.52$, 115.59. 117.61. 123.50. 126.41. 127.70. 133.64. 135.95.

\section{References and Notes}

1. For the reviews of Baylis-Hillman reaction and chemical transformations of the Baylis-Hillman adduct, see: (a) Basavaiah, D. Rao. A. T.: Satyanarayana. T. Chent Rer: 2003. 103. 811. (b) Ciganek. E.. Ed. In Organic Reactions: Paquette. L. A.. Ed.: Tohn Wiley \& Sons: New York. 1997: Vol. 51. pp 201-350. (c) Basavaiah. D.: Rao. P. D.: Hyma. R. S. Tetrohedhon 1996. 52. 8001 . (d) Kim. J. N.; Lee. K. Y. Cw7. Org. Chem. 2002, 6, 627. (e) Lee. K. Y; Gowrisankar, S.; Kim. J. N. Bull. Korean Chem. Soc. $2005,26,1481$, and further references cited therein.

2. For the chemical transformations of the Baylis-Hillman adducts of 2-titrobenzaldehydes. see: (a) Kim. J. N.: Lee. K. Y.: Kimn. H. S.: Kinn. T. Y. Org. Lett. 2000. 2. 343. (b) Lee. K. Y.: Kim. J. M.: Kinn. T. N. Bull. Korean Chent. Soc. 2002. 23. 1493. (c) Lee. K. Y.: Kim. J. N. Bull Korean Chem. Soc. 2002. 23. 939. (d) Lee, K. Y: Kim. J. M.: Kim. J. N. Tetrahedron 2003. 59, 385. (e) Lee, K. Y.; Kim. S. C.: Kim, J. N. Bull. Korean Chem. Soc. 2005 . 26, 1109.

3. For the chemical transformations of the Baylis-Hillman adducts of 2-titrobenzaldehydes. see: (a) Basavaiah. D: Reddy. R. M. Kumaragurubaran. N.: Sharada. D. S. Tetwahedon 20012. 58. 3693. (b) Familoni, O. B.; Kave, P. T.; Klass, P. J. J. Chem. Soc. Chem. Commm. 1998, 2563.

4. Synthesis indole derivatives from Baylis-Hillman adducts, see: (a) O'Dell. D. K.: Nicholas. K. M. Tetrahedron 2003. 59. 747. (b) O'Dell. D. K.: Nicholas. K. M. J. Org. Chem. 2003. 68. 6427. (c) Horn. C. R.; Perez, M. Synlett 2005, 1480 . (d) Pan, W: Dong. D.: Sun. S.: Liu, Q. Symlett 2006. 1090. (e) Yaday. J. S.: Reddy, B. V. S.: Basak. A. K.: Narsaiah. A. V.: Prabhakar. A.: Jagadeesh. B. Tetrahedron Lett. 2005. t6. 639. (f) Basavaiah. D.: Rao. A. J. Tetrahedron Lett. 2003. H4. 4365.

5. For the synthesis of indole derivatives. see: (a) Islam. M. S.: Brent1an1. C.: Wang. Q.: Hossain. M. M. J. Org. Chent. 2006. 71. 4675. (b) Penoni. A.: Volkmann. J.: Nicholas, K. M. Org. Lett. 2002, f, 699. (c) Stanovnik. B.: Tisler, M.: Carlock, J. T. Synthesis 1976, 754. (d) Mali, R. S.: Babu, K. N.: Jagtap. P. G. J. Chem. Res. (S) 1995. 114. (e) Belley. M.: Scheigetz. I.: Dube. P.: Dolman. S. Stmlet 2001. 222. (f) Somei. M.: Kizu. K.: Kunomoto. M.: Yamada. F. Chem. Phom. Bull 1985. 33. 3696. (g) Mali. R. S. Yadav, V. J. Sunthesis 1984. 862. (h) Akazome. M: Kondo, T. Watanabe. Y. J. Org. Chem 1994. 59. 3375. (i) Kondo. K.: Morohoshi. S.; Mitsuhashi. M.: Murakami. Y. Chem. Pham Bull. 1999. 47. 1227. (j) Mali. R. S.: Tilve. S. G.: Desai. V. G. J. Chem. Res. (S) 2000.8.

6. Shieh. W.-C.: Dell. S.: Bach. A.: Repic. O.: Blacklock. T. I. J. Org. Chem 2003, 68, 1954

7. Unpublished results.

8. (a) Kabalka, G. W: Varma. R. S. In Comprehensive Organic Smhesis: Trost. B. M.: Fleming. I.. Eds: Osford: Pergamon. 1991: Vol 8. pp 363-379. (b) Gowenlock. B. G: Richter-Addo. G. B. Chem. Rev 2004, 104, 3315. (c) Sawant, D.: Kumar, R: Maulik, P. R.: Kundu. B. Org. Lett. 2006. 8, 1525 . (d) Bates. D. K.: Li. K. J. Org. Chent. 2002. 67. 8662. 